\title{
Erratum to: Arnowitt-Deser-Misner representation and Hamiltonian analysis of covariant renormalizable gravity
}

\author{
Masud Chaichian $^{\mathrm{a}}$, Markku Oksanen ${ }^{\mathrm{b}}$, Anca Tureanu ${ }^{\mathrm{c}}$ \\ Department of Physics, University of Helsinki, P.O. Box 64, 00014 Helsinki, Finland
}

Received: 3 July 2011 / Published online: 13 September 2011

(C) Springer-Verlag / Società Italiana di Fisica 2011

\section{Erratum to: Eur. Phys. J. C (2011) 71:1657 \\ DOI 10.1140/epjc/s10052-011-1657-7}

\section{The source of error}

The idea of the notation convention was simple: denote tensors on the spacetime with the ${ }^{(4)}$ prefix and tensors that are tangent to the spatial hypersurface $\Sigma_{t}$ without the prefix. According to this convention it makes sense to denote the component of the Ricci tensor ${ }^{(4)} R_{\mu \nu}$ that is tangent to $\Sigma_{t}$ by $R_{\mu \nu}$. However, $R$ is already used to denote the intrinsic curvature of $\Sigma_{t}$. Since these two things are not the same, there is a misleading conflict in the notation.

\section{Erratum}

On page 4, the paragraph that contains (2.30)-(2.36) should be corrected as follows.

The Ricci tensor ${ }^{(4)} R_{\mu \nu}$ of spacetime can be decomposed as

$$
{ }^{(4)} R_{\mu \nu}={ }_{\perp}^{(4)} R_{\mu \nu}-{ }_{\perp}^{(4)} R_{\mu n} n_{\nu}-{ }_{\perp}^{(4)} R_{n \nu} n_{\mu}+{ }^{(4)} R_{n n} n_{\mu} n_{\nu},
$$

where we have defined

The online version of the original article can be found under doi:10.1140/epjc/s10052-011-1657-7.

\footnotetext{
a e-mail: masud.chaichian @helsinki.fi

b e-mail: markku.oksanen@helsinki.fi

c e-mail: anca.tureanu@helsinki.fi
}

$$
\begin{aligned}
&{ }_{\perp}^{(4)} R_{\mu \nu} \equiv g_{\mu}^{\rho}{ }_{\mu} g_{\nu}^{\sigma(4)} R_{\rho \sigma} \\
&= R_{\mu \nu}+K K_{\mu \nu}-2 K_{\mu \rho} K_{\nu}^{\rho}-\frac{1}{N} D_{\mu} D_{\nu} N \\
& \quad+\frac{1}{N} \mathcal{L}_{N n} K_{\mu \nu}, \\
&{ }_{\perp}^{(4)} R_{\mu n} \equiv g^{\rho}{ }_{\mu} n^{\nu(4)} R_{\rho \nu}=D_{\rho} K_{\mu}^{\rho}-D_{\mu} K, \\
&{ }_{\perp}^{(4)} R_{n v} \equiv g^{\rho}{ }_{\nu} n^{\mu(4)} R_{\mu \rho}=D_{\rho} K_{\nu}^{\rho}-D_{\nu} K \\
&{ }^{(4)} R_{n n} \equiv{ }^{(4)} R_{\mu \nu} n^{\mu} n^{\nu}=\frac{1}{2}\left(K^{2}-K_{i j} K^{i j}+R-{ }^{(4)} R\right) \\
&=K^{2}-K_{i j} K^{i j}-\nabla_{\mu}\left(n^{\mu} K\right)+\frac{1}{N} D^{i} D_{i} N .
\end{aligned}
$$

In (2.31) $R_{\mu \nu}$ is the Ricci tensor of the hypersurface $\Sigma_{t}$ and $\mathcal{L}_{N n}$ denotes the Lie derivative along $N n^{\mu}$. Note that for any tensor field $T$ that is tangent to $\Sigma_{t}, \mathcal{L}_{N n} T$ is also tangent to $\Sigma_{t}$. In (2.31)-(2.34) we have used the Gauss relation, the Ricci equation and the Codazzi relation, and in (2.34) the decomposition of ${ }^{(4)} R$ from (2.25) was also used. Hence the Einstein tensor can be decomposed:

$$
\begin{aligned}
{ }^{(4)} G_{\mu \nu} \equiv & { }^{(4)} R_{\mu \nu}-\frac{1}{2}{ }^{(4)} g_{\mu \nu}{ }^{(4)} R \\
= & R_{\mu \nu}+K K_{\mu \nu}-2 K_{\mu \rho} K_{\nu}^{\rho} \\
& -\frac{1}{N} D_{\mu} D_{\nu} N+\frac{1}{N} \mathcal{L}_{N n} K_{\mu \nu} \\
& -\frac{1}{2} g_{\mu \nu}\left(R+K_{i j} K^{i j}+K^{2}+2 \nabla_{n} K\right. \\
& \left.-\frac{2}{N} D^{i} D_{i} N\right) \\
& +\left(D_{\mu} K-D_{\rho} K^{\rho}{ }_{\mu}\right) n_{\nu}+n_{\mu}\left(D_{v} K-D_{\rho} K_{\nu}^{\rho}\right) \\
& +\frac{1}{2} n_{\mu} n_{v}\left(K^{2}-K_{i j} K^{i j}+R\right),
\end{aligned}
$$


where we have also used (2.29). Thus in the actions (2.13) and (2.14) we have

$$
\begin{aligned}
\partial^{\mu} \phi \partial^{\nu} \phi^{(4)} G_{\mu \nu}= & D^{i} \phi D^{j} \phi\left[R_{i j}+K K_{i j}-2 K_{i k} K_{j}^{k}\right. \\
& -\frac{1}{N} D_{i} D_{j} N+\frac{1}{N} \mathcal{L}_{N n} K_{i j} \\
& -\frac{1}{2} g_{i j}\left(R+K_{k l} K^{k l}+K^{2}\right. \\
& \left.\left.+2 \nabla_{n} K-\frac{2}{N} D^{k} D_{k} N\right)\right] \\
& +2\left(\nabla_{n} \phi\right) D^{i} \phi\left(D_{i} K-D^{j} K_{j i}\right) \\
& +\frac{1}{2}\left(\nabla_{n} \phi\right)^{2}\left(K^{2}-K_{i j} K^{i j}+R\right) .
\end{aligned}
$$

In the Hamiltonian analysis of Sect. 3. Hamiltonian formalism, the variables $N_{i}$ should be replaced by $N^{i}$, which are related by $N_{i}=g_{i j} N^{j}$. Consequently the following changes to the constraints of the theory are required.

For the case $z=3$ in Sect. 3.1, the momenta conjugate to the shift variables $N^{i}$ are written as $p_{i}$, which belong to the primary constraints (3.1) of the theory. The momentum constraint $\mathcal{H}_{3}^{i}$ is replaced with $\mathcal{H}_{i}^{3}=g_{i j} \mathcal{H}_{3}^{j}$ and accordingly redefined in (3.11) as

$\mathcal{H}_{i}^{3}=-2 g_{i j} D_{k} p^{j k}+D_{i} \zeta_{2} p_{\zeta_{2}}$.

The smeared momentum constraint $\Phi_{3}^{S}\left(\chi_{i}\right)$ is replaced with $\Phi_{S}^{3}\left(\chi^{i}\right)$ and redefined in (3.21) as

$\Phi_{S}^{3}\left(\chi^{i}\right)=\int \mathrm{d}^{3} \boldsymbol{x} \chi^{i} \mathcal{H}_{i}^{3} \approx 0$.

The Poisson bracket in (3.24) is corrected as

$\left\{\Phi_{S}^{3}\left(\chi^{i}\right), \Phi_{S}^{3}\left(\psi^{i}\right)\right\}=\Phi_{S}^{3}\left(\chi^{j} \partial_{j} \psi^{i}-\psi^{j} \partial_{j} \chi^{i}\right) \approx 0$.

Otherwise the presented algebra of constraints corresponds to the choice of variables $N^{i}$ and the associated momentum constraint $\mathcal{H}_{i}^{3}$. Indeed, if one uses $\mathcal{H}_{3}^{i}$ the Poisson bracket $\left\{\Phi_{3}^{S}\left(\chi_{k}\right), p^{i j}\right\}$ contains an additional term $-\chi^{(i} \mathcal{H}^{j)}$ compared to $\left\{\Phi_{S}^{3}\left(\chi^{k}\right), p^{i j}\right\}$, which was not intended.

Similar correction is applied to the case $z=4$ in Sect. 3.2. 